\title{
Rational Shi tableaux and the skew length statistic
}

\author{
Robin Sulzgruber If $^{\dagger}$ \\ ${ }^{1}$ Fakultät für Mathematik, Universität Wien, Austria
}

\begin{abstract}
We define two refinements of the skew length statistic on simultaneous core partitions. The first one relies on hook lengths and is used to prove a refined version of the theorem stating that the skew length is invariant under conjugation of the core. The second one is equivalent to a generalisation of Shi tableaux to the rational level of Catalan combinatorics. We prove that the rational Shi tableau is injective. Moreover we present a uniform definition of the rational Shi tableau for Weyl groups and conjecture injectivity in the general case.

Résumé: Nous définissons deux raffinements de la statistique de longueur gauche sur les partitions simultanément coeur. Le premier repose sur les longueurs d'équerre et est utilisé pour prouver une version plus fine du théorème d'invariance de la longueur gauche par conjugaison du coeur. Le second est équivalent à une généralisation des tableaux de Shi au niveau rationnel de la combinatoire de Catalan. Nous démontrons que le tableau de Shi rationnel est injectif. De plus nous présentons une définition uniforme du tableau de Shi rationnel pour les groupes de Weyl, et conjecturons l'injectivité dans le cas général.
\end{abstract}

Keywords. affine permutations, cores, Dyck paths, Shi arrangement, skew length

The skew length is a statistic on simultaneous core partitions, which counts the number of certain cells in the Young diagram of the core. It was invented by Armstrong [AHJ14] who used it to give a combinatorial formula for the rational $q, t$-Catalan numbers (see also [GM13, ALW15])

$$
C_{n, p}(q, t)=\sum_{\kappa \in \mathfrak{C}_{n, p}} q^{\ell(\kappa)} t^{(n-1)(p-1) / 2-\operatorname{skl}(\kappa)} .
$$

Here $n$ and $p$ are relatively prime, $\mathfrak{C}_{n, p}$ denotes the set of $n, p$-cores, $\ell(\kappa)$ is the length of $\kappa$ and $\operatorname{skl}(\kappa)$ denotes the skew length (see Section 1 for all definitions). The intriguing property of these polynomials is the apparent symmetry $C_{n, p}(q, t)=C_{n, p}(t, q)$, for which there is no proof except in very special cases.

The skew length was further studied in [Xin15] and [CDH15]. For coprime $n$ and $p$ it was proven that $\operatorname{skl}(\kappa)$ is invariant under conjugation of the core and independent of whether $\kappa$ is viewed as an $n, p$-core or as a $p, n$-core. The first main contribution of our paper is a refinement of the skew length statistic in terms of the multiset of hook lengths of certain cells of the core. We obtain a refined version of the two results mentioned above in Theorem 1.3 providing a conceptual explanation for these phenomena. Moreover our

\footnotetext{
†Email: robin.sulzgruber@univie.ac.at. Research supported by the Austrian Science Fund (FWF), grant S50-N15 in the framework of the Special Research Program "Algorithmic and Enumerative Combinatorics" (SFB F50).
} 
method of proof relies on induction and does not use rational Dyck paths. This has the advantage that we do not need to assume that $n$ and $p$ are relatively prime. Theorem 1.3 therefore extends to previously untreated cases.

Shi tableaux [FTV11] encode the dominant regions of the ( $m$-extended) Shi arrangement, which are counted by the Fuß-Catalan numbers. The Shi arrangement and its regions are intimately related to the affine symmetric group. In [GMV14] Gorsky, Mazin and Vazirani define the so called $p$-stable affine permutations, which can be seen as a generalisation of the regions of the Shi arrangement to the rational level of Catalan combinatorics. In Section 2 we define rational Shi tableaux, which encode dominant $p$-stable affine permutations. Our main result concerning rational Shi tableaux is Theorem 2.8, asserting that each dominant $p$-stable affine permutation is uniquely determined by its Shi tableau. Theorem 2.8 is especially interesting in view of the fact that the rational Pak-Stanley labelling [GMV14] can be obtained by taking the row-sums of the rational Shi tableau.

The Shi arrangement can be defined for any irreducible crystallographic root system $\Phi$. Furthermore, $p$-stable affine Weyl group elements have been considered in [Thi15]. We provide a uniform definition of the rational Shi tableaux of dominant $p$-stable elements of the affine Weyl group of $\Phi$. We conjecture that dominant $p$-stable Weyl group elements are determined uniquely by their Shi tableaux (Conjecture 2.7p).

In Section 3 we tie our previous results together by relating dominant $p$-stable affine permutations to rational Dyck paths and simultaneous cores. This is achieved using the Anderson bijection of [GMV14]. We show that the rational Shi tableau of a dominant $p$-stable affine permutation can be computed from the corresponding rational Dyck path as the codinv tableau of the path (Theorem 3.2). The codinv statistic on rational Dyck paths, that is, the sum of the entries of the codinv tableau, corresponds naturally to the skew length statistic on cores (Corollary 3.4). Hence we find that rational Shi tableaux can in fact be regarded as another refinement of the skew length statistic. The contents of Section 3 and in particular the connection between rational Shi tableaux and the zeta map on rational Dyck paths [GM13, ALW15] (Theorem 3.3] are the main tools used in the proof of Theorem 2.8

This is an extended abstract and some proofs have been omitted. A preprint of the full version is available [Sul15].

\section{Skew length and conjugation}

Throughout this section $n$ and $p$ are positive integers (not necessarily coprime!). For any positive integer $z$ we set $[z]=\{1, \ldots, z\}$. A partition $\lambda$ is a weakly decreasing sequence $\lambda_{1} \geq \lambda_{2} \geq \cdots \geq \lambda_{\ell}>0$ of positive integers. The number of parts or summands $\lambda_{i}$ is called the length of the partition and is denoted by $\ell(\lambda)$. The sum $\sum_{i} \lambda_{i}$ is called the size of $\lambda$. A partition is often identified with its Young diagram $\left\{(i, j): i \in[\ell(\lambda)], j \in\left[\lambda_{i}\right]\right\}$. We call the elements of the Young diagram cells of the partition. The conjugate of the partition $\lambda$ is the partition $\lambda^{\prime}$ where $\lambda_{i}^{\prime}=\#\left\{j: \lambda_{j} \geq i\right\}$. Equivalently, if we view partitions as Young diagrams then $(j, i) \in \lambda^{\prime}$ if and only if $(i, j) \in \lambda$. The hook length of a cell of $\lambda$ is defined as $h_{\lambda}(i, j)=\lambda_{i}-j+\lambda_{j}^{\prime}-i+1$. A partition $\kappa$ is called $n$-core if no cell of $\kappa$ has hook length $n$. A partition is called $n, p$-core if it is both an $n$-core and a $p$-core. We denote the set of $n$-core partitions by $\mathfrak{C}_{n}$ and the set of simultaneous $n, p$-cores by $\mathfrak{C}_{n, p}$. Clearly $\lambda \in \mathfrak{C}_{n}$ if and only if $\lambda^{\prime} \in \mathfrak{C}_{n}$.

Let $\lambda$ be a partition. Given the hook length $h$ of a cell $x$ in the top row of $\lambda$ we denote by $H^{c}(h)$ the set of hook lengths of cells in the same column as $x$. Given the hook length $h$ of a cell $x$ in the first column of $\lambda$ we denote by $H^{r}(h)$ the set of hook lengths of cells in the same row as $x$. If $h$ is not the hook length 


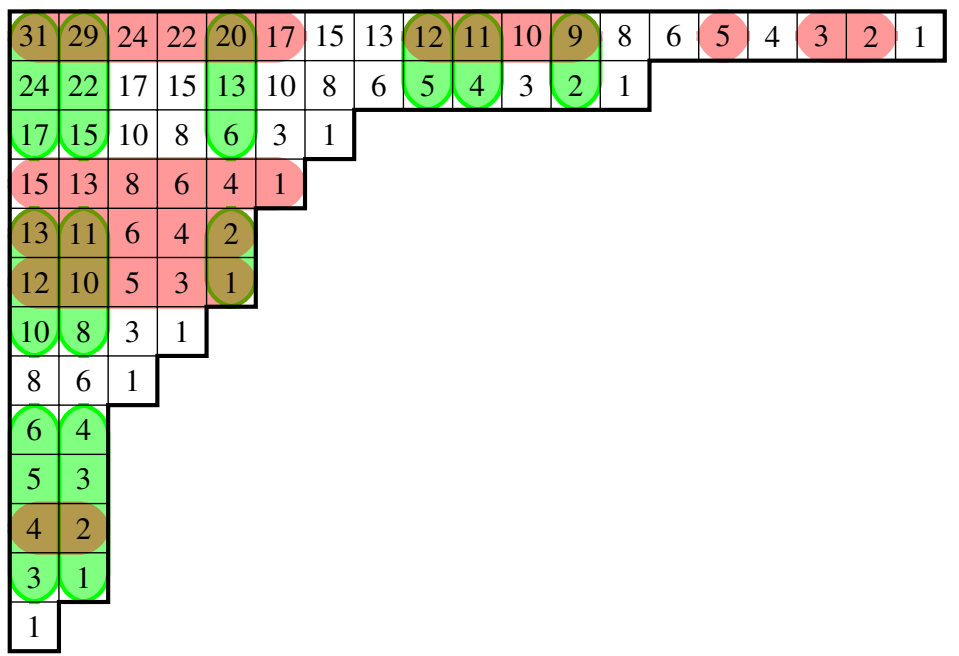

Fig. 1: A simultaneous core $\kappa \in \mathfrak{C}_{7,16}$ with the multisets $H_{7,16}(\kappa)$ in red and $H_{16,7}(\kappa)$ in green.

of a suitable cell, set $H^{r}(h)=\emptyset$ resp. $H^{c}(h)=\emptyset$. For example, in Figure 1 we have $H^{c}(12)=\{12,5\}$ and $H^{r}(12)=\{12,10,5,3,1\}$ and $H^{r}(11)=\emptyset$.

A subset $A \subseteq \mathbb{Z}$ is called abacus if there exist integers $a, b \in \mathbb{Z}$ such that $z \in A$ for all $z$ with $z<a$, and $z \notin A$ for all $z$ with $z>b$. We call the elements of $A$ beads and the elements of $\mathbb{Z}-A$ gaps. An abacus is normalised if zero is a gap and there are no negative gaps. An abacus is balanced if the number of positive beads equals the number of nonpositive gaps. An abacus $A$ is $n$-flush if $z-n \in A$ for all $z \in A$. The theorem below is a version of the classical result that $n$-cores correspond to abacus diagrams that are $n$-flush (see for example [JK81, 2.7.13]). Let $\kappa \in \mathfrak{C}_{n}$ be an $n$-core with maximal hook length $m$, that is, $m$ is the hook length of the top left corner. Define $\alpha(\kappa)=\left\{\kappa_{i}+1-i: i \geq 1\right\}$ and $\beta(\kappa)=H^{c}(m) \cup\{z \in \mathbb{Z}: z<0\}$, where by convention $\kappa_{i}=0$ when $i>\ell(\kappa)$. Note that $\beta(\kappa)=\{z+\ell(\kappa)-1: z \in \alpha(\kappa)\}$.

Theorem 1.1 The map $\alpha$ is a bijection between $n$-cores and balanced $n$-flush abaci. The map $\beta$ is a bijection between $n$-cores and normalised $n$-flush abaci.

For our purposes in this section we need the following simple consequence of Theorem 1.1 .

Lemma 1.2 Let $\kappa \in \mathfrak{C}_{n}$ be an $n$-core and $z \geq 0$. Then $z+n \in H^{r}(h)$ implies $z \in H^{r}(h)$, and $z+n \in H^{c}(h)$ implies $z \in H^{c}(h)$.

In fact, the set of $n$-cores is characterised by the property that $z+n \in H^{c}(m)$ implies $z \in H^{c}(m)$ for all $z \geq 0$, where $m$ is the maximal hook length of $\kappa$.

Let $\kappa \in \mathfrak{C}_{n, p}$ be a simultaneous core with maximal hook length $m$. Moreover choose an element $h \in H^{c}(m)$. We call the row of $\kappa$ whose leftmost cell has hook length $h$ an $n$-row (resp. $p$-row) if $h+n \notin H^{c}(m)$ (resp. $h+p \notin H^{c}(m)$ ). Similarly, given $h \in H^{r}(m)$ we call the column whose top cell has hook length $h$ an $n$-column (resp. $p$-column) if $h+n \notin H^{r}(m)$ (resp. $h+p \notin H^{r}(m)$ ). Denote by 
$H_{n, p}(\kappa)$ the multiset of hook lengths of cells that are contained both in an $n$-row and in a $p$-column. In Figure 1 the leftmost hook lengths of the 7-rows are 31, 15,13,12 and 4, and the top hook lengths of the 7-columns are 31,29, 20,12, 11 and 9 .

This section's main result is a surprising symmetry property of the multiset $H_{n, p}(\kappa)$.

Theorem 1.3 Let $\kappa \in \mathfrak{C}_{n, p}$ be an $n, p$-core. Then $H_{n, p}(\kappa)=H_{p, n}(\kappa)$.

Proof: We prove the claim by induction on the size of $\kappa$. Denote by $\bar{\kappa}$ the partition obtained from $\kappa$ by deleting the first column. Clearly $\bar{\kappa} \in \mathfrak{C}_{n, p}$ and we may assume that $H_{n, p}(\bar{\kappa})=H_{p, n}(\bar{\kappa})$.

Let $m$ denote the maximal hook length in $\kappa$. Note that each $n$-row of $\bar{\kappa}$ is an $n$-row of $\kappa$. The only $p$ column of $\bar{\kappa}$ that is not a $p$-column of $\kappa$ has maximal hook length $m-p$. Thus there exist sets $A \subseteq H^{c}(m)$ and $B \subseteq H^{c}(m-p)$ with $H_{n, p}(\kappa)=\left(H_{n, p}(\bar{\kappa}) \cup A\right)-B$. Similarly $H_{p, n}(\kappa)=\left(H_{p, n}(\bar{\kappa}) \cup C\right)-D$ for some sets $C \subseteq H^{c}(m)$ and $D \subseteq H^{c}(m-n)$. It suffices to show that $A-B=C-D$ and $B-A=D-C$.

Suppose $z \in A$ but $z \notin B$. Then $z \in H^{c}(m)$ and $z+n \notin H^{c}(m)$. On the one hand we obtain $z \notin H^{c}(m-n)$ and $z \notin D$. It follows that $A \cap D=\emptyset$. On the other hand we obtain $z+n+p \notin H^{c}(m)$ and therefore $z+n \notin H^{c}(m-p)$. Since $z \notin B$ this implies $z \notin H^{c}(m-p)$ and consequently $z+p \notin H^{c}(m)$. We obtain $z \in C$. Therefore $A-B \subseteq C-D$, and $A-B=C-D$ by symmetry.

Conversely suppose $z \in B$ but $z \notin A$. By symmetry we have $B \cap C=\emptyset$ and $z \notin C$. On the other hand $z+n \notin H^{c}(m-p)$ implies $z+n+p \notin H^{c}(m)$ and thus $z+p \notin H^{c}(m-n)$. Moreover $z \in H^{c}(m-p)$ implies $z+p \in H^{c}(m)$ and therefore $z \in H^{c}(m)$. Since $z \notin A$ we obtain $z+n \in H^{c}(m)$ and $z \in H^{c}(m-n)$. We conclude that $z \in D$ and the proof is complete.

The skew length $\operatorname{skl}(\kappa)$ of an $n, p$-core was defined by Armstrong, Hanusa and Jones [AHJ14] as the number of cells that are contained in an $n$-row of $\kappa$ and have hook length less than $p$. The set $H_{n, p}(\kappa)$ allows for a new equivalent definition.

Proposition 1.4 Let $\kappa \in \mathfrak{C}_{n, p}$ be an $n, p$-core. Then $\operatorname{skl}(\kappa)=\# H_{n, p}(\kappa)$.

From Theorem 1.3 we immediately recover two results that were recently proven by Guoce Xin [Xin15], and independently by Ceballos, Denton and Hanusa [CDH15] when $n$ and $p$ are relatively prime.

Corollary 1.5 The skew length of an n, $p$-core is independent of the order of $n$ and $p$.

Corollary 1.6 Let $\kappa \in \mathfrak{C}_{n, p}$ be an $n, p$-core with conjugate $\kappa^{\prime}$. Then $\operatorname{skl}(\kappa)=\operatorname{skl}\left(\kappa^{\prime}\right)$.

Indeed, with our alternative definition of skew length given in Proposition 1.4 the statements of Corollaries 1.5 and 1.6 are identical. We close this section by stating two equivalent conjectural properties of the multiset $H_{n, p}(\kappa)$ that need further investigation.

Conjecture 1.7 Let $n$ and $p$ be relatively prime, and $\kappa \in \mathfrak{C}_{n, p}$. Then the hook length of each cell of $\kappa$ appears in $H_{n, p}(\kappa)$ with multiplicity at least one.

Conjecture 1.8 Let $n$ and $p$ by relatively prime, $z \geq 0$ and $\kappa \in \mathfrak{C}_{n, p}$. Then $z+n \in H_{n, p}(\kappa)$ implies $z \in H_{n, p}(\kappa)$. Equivalently also $z+p \in H_{n, p}(\kappa)$ implies $z \in H_{n, p}(\kappa)$. 


\section{Rational Shi tableaux}

In the beginning of this section we recall some facts about root systems and Weyl groups. For further details we refer the reader to [Hum90]. Let $\Phi$ be an irreducible crystallographic root system with ambient space $V$, positive system $\Phi^{+}$and simple system $\Delta=\left\{\sigma_{1}, \ldots, \sigma_{r}\right\}$. Any root $\alpha \in \Phi$ can be written as a unique integer linear combination $\alpha=\sum_{i=1}^{r} c_{i} \sigma_{i}$, where all coefficients $c_{i}$ are nonnegative if $\alpha \in \Phi^{+}$, or all coefficients are nonpositive if $\alpha \in-\Phi^{+}$. We define the height of the root $\alpha$ by $\operatorname{ht}(\alpha)=\sum_{i=1}^{r} c_{i}$. Thereby ht $(\alpha)>0$ if and only if $\alpha \in \Phi^{+}$, and ht $(\alpha)=1$ if and only if $\alpha \in \Delta$. Moreover there exists a unique highest root $\tilde{\alpha}$ such that $\operatorname{ht}(\tilde{\alpha}) \geq \operatorname{ht}(\alpha)$ for all $\alpha \in \Phi$. The Coxeter number of $\Phi$ can be defined as $h=\operatorname{ht}(\tilde{\alpha})+1$.

Let $\delta$ be a formal variable. We define the set of affine roots as $\widetilde{\Phi}=\{\alpha+k \delta: \alpha \in \Phi, k \in \mathbb{Z}\}$. The height of an affine root is given by $\operatorname{ht}(\alpha+k \delta)=\mathrm{ht}(\alpha)+k h$. The sets of positive and simple affine roots are defined as $\widetilde{\Phi}^{+}=\Phi^{+} \cup\{\alpha+k \delta \in \widetilde{\Phi}: \alpha \in \Phi, k>0\}$ and $\widetilde{\Delta}=\Delta \cup\{-\tilde{\alpha}+\delta\}$. Thus $\alpha+k \delta \in \widetilde{\Phi}^{+}$if and only if $\mathrm{ht}(\alpha+k \delta)>0$, and $\alpha+k \delta \in \widetilde{\Delta}$ if and only if ht $(\alpha+k \delta)=1$.

The Coxeter arrangement $\operatorname{Cox}(\Phi)$ consists of all hyperplanes of the form $H_{\alpha}=\{x \in V:\langle x, \alpha\rangle=0\}$ for $\alpha \in \Phi$. Its regions, that is, the connected components of $V-\bigcup_{\alpha \in \Phi} H_{\alpha}$, are called chambers. We define the dominant chamber as

$$
C=\{x \in V:\langle x, \alpha\rangle>0 \text { for all } \alpha \in \Delta\} .
$$

The Weyl group $W$ of $\Phi$ is the group of linear automorphisms of $V$ generated by all reflections in a hyperplane in $\operatorname{Cox}(\Phi)$. The Weyl group acts simply transitively on the chambers. Thus identifying the identity $e \in W$ with the dominant chamber, each chamber corresponds to a unique Weyl group element.

The affine arrangement $\operatorname{Aff}(\Phi)$ consists of all hyperplanes of the form $H_{\alpha, k}=\{x \in V:\langle x, \alpha\rangle=k\}$, where $\alpha \in \Phi$ and $k \in \mathbb{Z}$. Its regions are called alcoves. We define the fundamental alcove as

$$
A_{\circ}=\{x \in V:\langle x, \alpha\rangle>0 \text { for all } \alpha \in \Delta \text { and }\langle x, \tilde{\alpha}\rangle<1\} .
$$

The affine Weyl group $\widetilde{W}$ of $\Phi$ is the group of affine transformations of $V$ that is generated by all reflections in a hyperplane in $\operatorname{Aff}(\Phi)$. The affine Weyl group acts simply transitively on the set of alcoves. By identifying the identity $e \in \widetilde{W}$ with the fundamental alcove, every alcove corresponds to a unique element of $\widetilde{W}$. An element $\omega \in \widetilde{W}$ is called dominant if and only if the alcove $\omega\left(A_{\circ}\right)$ is contained in the dominant chamber $C$.

Given a root $\alpha \in \Phi$ we define its coroot as $\alpha^{\vee}=2 \alpha /\langle\alpha, \alpha\rangle$. The coroot lattice is the integer span of all coroots $\check{Q}=\sum_{\alpha \in \Phi} \mathbb{Z}^{\vee} \subseteq V$. For each $q \in \check{Q}$ the translation $t_{q}: V \rightarrow V$ defined by $t_{q}(x)=x+q$ for all $x \in V$ is an element of the affine Weyl group. Identifying $Q$ with its translation group we obtain $\widetilde{W}=W \ltimes \check{Q}$. Note that if $\omega \in \widetilde{W}$ is dominant and $\omega=t_{q} s$, where $q \in \check{Q}$ and $s \in W$, then in particular $q$ lies in the closure of the dominant chamber. Thus $\langle\alpha, q\rangle \geq 0$ for each positive root $\alpha \in \Phi^{+}$.

Fix a root system of type $A_{n-1}$ by declaring the roots, positive roots and simple roots to be

$$
\Phi=\left\{e_{i}-e_{j}: i, j \in[n], i \neq j\right\}, \quad \Phi^{+}=\left\{e_{i}-e_{j}: i, j \in[n], i<j\right\}, \quad \Delta=\left\{e_{i}-e_{i+1}: i \in[n-1]\right\} .
$$

We now describe a combinatorial model for the affine Weyl group of type $A_{n-1}$. A detailed exposition is found in [BB05, Sec. 8.3]. The affine symmetric group $\widetilde{\mathfrak{S}}_{n}$ is the group of bijections $\omega: \mathbb{Z} \rightarrow \mathbb{Z}$ such 
that $\omega(i+n)=\omega(i)+n$ for all $i \in \mathbb{Z}$ and $\omega(1)+\cdots+\omega(n)=n(n+1) / 2$. Such a bijection $\omega$ is called affine permutation. Each affine permutation is uniquely determined by its window $[\omega(1), \omega(2), \ldots, \omega(n)]$. The group $\widetilde{\mathfrak{S}}_{n}$ has a set of generators called simple transpositions given by $s_{i}=[1, \ldots, i+1, i, \ldots, n]$ for $i \in$ $[n-1]$ and $s_{n}=[0,2, \ldots, n-1, n+1]$. The affine symmetric group and the set of simple transpositions form a Coxeter system isomorphic to the the affine Weyl group of type $A_{n-1}$. The symmetric group $\mathfrak{S}_{n}$ can be seen as the subgroup of $\widetilde{\mathfrak{S}}_{n}$ consisting of all affine permutations whose window is a permutation of $[n]$.

The affine symmetric group possesses an involutive automorphism owing to the symmetry of the Dynkin diagram of type $A_{n-1}$. Set $s_{i}^{*}=s_{n-i}$ for $i \in[n-1]$ and $s_{n}^{*}=s_{n}$. This correspondence extends to an automorphism $\omega \mapsto \omega^{*}$ on $\widetilde{\mathfrak{S}}_{n}$, where $\omega^{*}$ is obtained by replacing all instances of $s_{i}$ in any expression of $\omega$ in terms of the simple transpositions by $s_{i}^{*}$. The involutive automorphism has a simple explicit description in window notation and fulfils many desirable properties.

Lemma 2.1 (i) Let $\omega \in \widetilde{\mathfrak{S}}_{n}$ be an affine permutation and $i \in \mathbb{Z}$. Then $w^{*}(i)=1-\omega(1-i)$. In particular the window of $\omega^{*}$ is given by $[n+1-\omega(n), \ldots, n+1-\omega(1)]$.

(ii) The involutive automorphism preserves $\mathfrak{S}_{n}$, translations, and dominant affine permutations.

Let $\omega \in \widetilde{\mathfrak{S}}_{n}$ be an affine permutation. An affine inversion of $\omega$ is a pair $(i, j) \in[n] \times \mathbb{N}$ such that $i<j$ and $\omega(i)>\omega(j)$. For $i, j \in[n]$ with $i<j$ define

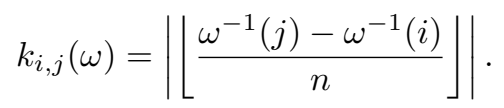

The numbers $k_{i, j}(\omega)$ where first considered by Shi [Shi86, Lem. 4.2.2]. We arrange them in a staircase tableau as in Figure 3

Lemma 2.2 (Compare to [BB05, Prop. 8.3.1]) Let $\omega \in \widetilde{\mathfrak{S}}_{n}$ be an affine permutation.

(i) Then the number of affine inversions of $\omega$ is given by $\sum_{i, j} k_{i, j}(\omega)$.

(ii) If $\omega$ is dominant then $k_{i, j}(\omega)$ equals the number of affine inversions $(a, b)$ of $\omega$, such that $\omega(a) \equiv i$ and $\omega(b) \equiv j$ modulo $n$.

Motivated by Lemma 2.2 we call the collection of numbers $k_{i, j}(\omega)$ for $i, j \in[n]$ with $i<j$ the inversion table of $\omega \in \widetilde{\mathfrak{S}}_{n}$. A simple computation reveals the effect of the involutive automorphism on the inversion table.

Proposition 2.3 Let $\omega \in \widetilde{\mathfrak{S}}_{n}$ be an affine permutation. Then the inversion table of $\omega^{*}$ is the transpose of the inversion table of $\omega$. That is $k_{n+1-j, n+1-i}\left(\omega^{*}\right)=k_{i, j}(\omega)$ for all $i, j \in[n]$ with $i<j$.

There is a natural way to generalise the inversion table to affine Weyl groups. Let $\Phi$ be an irreducible crystallographic root system with affine Weyl group $\widetilde{W}$. Let $\omega \in \widetilde{W}$ have the decomposition $\omega=t_{q} s$, where $q \in \check{Q}$ and $s \in W$. The affine Weyl group acts on the set of affine roots by

$$
\omega \cdot(\alpha+k \delta)=s \cdot \alpha+(k-\langle q, s \cdot \alpha\rangle) \delta .
$$

A positive affine root $\alpha+k \delta \in \widetilde{\Phi}^{+}$is an affine inversion of $\omega$ if $\omega \cdot(\alpha+k \delta) \in-\widetilde{\Phi}^{+}$. We denote the set of affine inversions of $\omega$ by $\operatorname{Inv}(\omega)=\widetilde{\Phi}^{+} \cap \omega^{-1} \cdot\left(-\widetilde{\Phi}^{+}\right)$. For $\omega \in \widetilde{W}$ and a positive root $\alpha \in \Phi^{+}$define

$$
k_{\alpha}(\omega)=\#\left(\operatorname{Inv}\left(\omega^{-1}\right) \cap\{ \pm \alpha+k \delta: k \in \mathbb{Z}\}\right) .
$$


Proposition 2.4 Let $i, j \in[n]$ with $i<j$ and $\omega \in \widetilde{\mathfrak{S}}_{n}$. Then $k_{i, j}(\omega)=k_{e_{i}-e_{j}}(\omega)$.

Note that two distinct elements of $\widetilde{\mathfrak{S}}_{n}$ may well have the same inversion table. However, we achieve a bijective correspondence between permutations and tableaux if we restrict ourselves to dominant affine permutations. We shall see that in case of the rational Shi tableau, which also counts certain inversions of affine permutations, the appropriate set is even smaller, in fact finite.

Let $p$ be a positive integer that is relatively prime to $n$. An affine permutation $\omega \in \widetilde{\mathfrak{S}}_{n}$ is called $p$-stable if $\omega(i)<\omega(i+p)$ for all $i \in \mathbb{Z}$. The set of $p$-stable affine permutations in $\widetilde{\mathfrak{S}}_{n}$, denoted by $\widetilde{\mathfrak{S}}_{n}^{p}$, was first considered by Gorsky, Mazin and Vazirani [GMV14]. As a consequence of Lemma 2.1] we see that the involutive automorphism $\omega \mapsto \omega^{*}$ preserves $\widetilde{\mathfrak{S}}_{n}^{p}$. If $p=m n+1$ then $\widetilde{\mathfrak{S}}_{n}^{p}$ corresponds to the set of minimal alcoves of the regions of the $m$-extended Shi arrangement. If $p=m n-1$ then $\widetilde{\mathfrak{S}}_{n}^{p}$ is related to the bounded regions of the $m$-extended Shi arrangement. Thus $p$-stable affine permutations should be viewed as a "rational" analogue of the regions of the Shi arrangement.

Let $\omega \in \widetilde{\mathfrak{S}}_{n}^{p}$ be a dominant $p$-stable affine permutation. Let $\omega^{-1}=t_{q} s$, where $q \in \check{Q}$ and $s \in \mathfrak{S}_{n}$, and $p=m n+r$ with $r \in[n-1]$. We define the rational Shi tableau of $\omega$ as the collection of integers

$$
t_{i, j}^{p}(\omega)= \begin{cases}\min \left(k_{i, j}(\omega), m\right) & \text { if } r+s(i)<s(j) \text { or } s(i)+r-n<s(j)<s(i) \\ \min \left(k_{i, j}(\omega), m+1\right) & \text { otherwise, }\end{cases}
$$

where $i, j \in[n]$ such that $i<j$.

One can show that $k_{i, j}(\omega) \leq m$ whenever $s(j)-s(i) \in\{r-n, r\}$. As a consequence $t_{i, j}^{p}(\omega)=$ $\min \left(k_{i, j}(\omega), m\right)$ if $p=m n+1$, and $t_{i, j}^{p}(\omega)=\min \left(k_{i, j}(\omega), m+1\right)$ if $p=m n+(n-1)$. For $p=m n+1$ we recover the Shi tableau of Fishel, Tzanaki and Vazirani [FTV11]

The rational Shi tableau behaves similarly to the inversion table under the involutive automorphism.

Proposition 2.5 Let $\omega \in \widetilde{\mathfrak{S}}_{n}^{p}$ be a dominant p-stable affine permutation. Then the rational Shi tableau of $\omega^{*}$ is the transpose of the rational Shi tableau of $\omega$. That is, $t_{n+1-j, n+1-i}^{p}\left(\omega^{*}\right)=t_{i, j}^{p}(\omega)$ for all $i, j \in[n]$ with $i<j$.

The rational Shi tableau generalises naturally to affine Weyl groups. Let $\Phi$ be an irreducible crystallographic root system, and $p$ be relatively prime to the Coxeter number $h$ of $\Phi$. Let $\widetilde{\Phi}_{p}$ denote the set of affine roots of height $p$. In [Thi15, Sec. 8] Thiel defines $p$-stable affine Weyl group elements as the set $\widetilde{W}^{p}=\left\{\omega \in \widetilde{W}: \omega \cdot \widetilde{\Phi}_{p} \subseteq \widetilde{\Phi}^{+}\right\}$. Let $\alpha \in \Phi^{+}$be a positive root and $\omega \in \widetilde{W}^{p}$ a dominant $p$-stable element of the affine Weyl group. Define

$$
t_{\alpha}^{p}(\omega)=\#\left(\omega \cdot\left(-\widetilde{\Phi}_{<p}^{+}\right) \cap\{-\alpha+k \delta: k \geq 1\}\right)
$$

where $\widetilde{\Phi}_{<p}^{+}$denotes the set of positive affine roots with height less than $p$. The rational Shi tableau of $\omega$ is the collection of numbers $t_{\alpha}^{p}(\omega)$ for $\alpha \in \Phi^{+}$.

Proposition 2.6 Let $n, p$ be positive coprime integers, $i, j \in[n]$ with $i<j$, and $\omega \in \widetilde{\mathfrak{S}}_{n}^{p}$ be a dominant $p$-stable affine permutation. Then $t_{i, j}^{p}(\omega)=t_{e_{i}-e_{j}}^{p}(\omega)$, which counts the number of affine inversions $(a, b)$ of $\omega$ such that $b<a+p$, and $\omega(a) \equiv i$ and $\omega(b) \equiv j$ modulo $n$.

The following is the main conjecture of this paper. 
Conjecture 2.7 Let $\Phi$ be an irreducible crystallographic root system with affine Weyl group $\widetilde{W}$ and Coxeter number $h$, and let $p$ be a positive integer relatively prime to $h$. Then each dominant $p$-stable element of the affine Weyl group is determined uniquely by its rational Shi tableau.

Conjecture 2.7 is known in to be true in the Fuß-Catalan case where $p=m n+1$. In the next section we exploit the connections of the affine symmetric group to the combinatorics of rational Dyck paths to prove the conjecture if $\Phi$ is of type $A_{n-1}$. That is, we prove the following theorem. (The proof is found at the end of Section 3)

Theorem 2.8 Let $n, p$ be two positive coprime integers. Then each dominant p-stable affine permutation in $\widetilde{\mathfrak{S}}_{n}$ is determined uniquely by its rational Shi tableau.

The sum of the entries of the Shi tableau generalises the height statistic used by Stump in [Stu10, Conj. 3.14]. Thus the $q$-Fuß-Catalan numbers proposed therein are generalised by the polynomials

$$
C_{\Phi, p}(q)=\sum_{\omega} \prod_{\alpha \in \Phi^{+}} q^{t_{\alpha}^{p}(\omega)}
$$

the sum being taken over all dominant $p$-stable elements of the affine Weyl group, after a suitable transformation. To be precise we have $q^{h m r / 2} \mathrm{Cat}^{(m)}\left(W ; q^{-1}\right)=C_{\Phi, h m+1}(q)$, where $r$ is the rank of $\Phi$.

Pak and Stanley [Sta96] found a bijection between the regions of the ( $m$-extended) Shi arrangement of type $A_{n-1}$ and the set of (m-)parking functions of length $n$. Gorsky, Mazin and Vazirani [GMV14, Def. 3.8] generalised this bijection to a map from $\widetilde{\mathfrak{S}}_{n}^{p}$ to the set of rational parking functions. Their rational Pak-Stanley labelling $f(\omega)$ is defined by

$$
f_{i}(\omega)=\#\{(a, b) \in[n] \times \mathbb{N}: a<b<a+p, \omega(a)>\omega(b) \text { and } \omega(b) \equiv i \text { modulo } n\},
$$

where $i \in[n]$. If $\omega$ is a dominant $p$-stable affine permutation then the Pak-Stanley labelling is obtained by taking the row-sums of the Shi tableau of $\omega$. That is, $f_{j}(\omega)=\sum_{i=1}^{j-1} t_{i, j}^{p}(\omega)$. Consequently the dual Pak-Stanley labelling, which we define by $f_{i}^{*}(\omega)=f_{i}\left(\omega^{*}\right)$, is obtained by taking the column-sums of the Shi tableau of $\omega$. That is, $f_{i}^{*}(\omega)=\sum_{j=n-i+2}^{n} t_{n-i+1, j}^{p}(\omega)$.

Partly the motivation for studying rational Shi tableaux comes from the fact that in view of the previous paragraph they can be regarded as an intermediate step between dominant $p$-stable affine permutations and their image under the Pak-Stanley labelling. If one replaces a dominant $p$-stable affine permutation $\omega$ by its rational Shi tableau then some information is apparently lost, since not all inversions of $\omega$ are taken into account. Moving on to the Pak-Stanley labelling $f(\omega)$ gives up even more information on the nature of these inversions. Nevertheless there seems to be just enough information left to determine $\omega$ uniquely. While the injectivity of the Pak-Stanley labelling on the set of all $p$-stable affine permutations remains an open problem [GMV14, Conj. 1.4], it follows from the work of Williams [Wil15] that the Pak-Stanley labelling is injective on the set of dominant $p$-stable affine permutations. In view of Theorem 2.8 it is an interesting question whether Shi tableaux can offer new insights regarding this problem.

Another major open problem concerning the Pak-Stanley labelling is to find an analogous labelling for different affine Weyl groups. Notably also this problem can be solved for rational Shi tableaux (recall the definition in (4)).

To conclude this section we want to connect the world of cores and abaci to dominant affine permutations. This connection is achieved by the next theorem, which follows essentially from the work of Lascoux [Las01]. Let $\omega \in \widetilde{\mathfrak{S}}_{n}$ be a dominant affine permutation, and define $\gamma(\omega)=\{z \in \mathbb{Z}: \omega(z) \leq 0\}$. 


\begin{tabular}{c|c|c|c|c|c|c|c|c|c|c|c|c|c|c|c|c|c|c|}
112 & 105 & 98 & 91 & 84 & 77 & 70 & 63 & 56 & 49 & 42 & 35 & 28 & 21 & 14 & 7 & 0 \\
96 & 89 & 82 & 75 & 68 & 61 & 54 & 47 & 40 & 33 & 26 & 19 & 12 & 5 & -2 & -9 & -16 \\
\hline 80 & 73 & 66 & 59 & 52 & 45 & 38 & 31 & 24 & 17 & 10 & 3 & -4 & -11 & -18 & -25 & -32 \\
64 & 57 & 50 & 43 & 36 & 29 & 22 & 15 & 8 & 1 & -6 & -13 & -20 & -27 & -34 & -41 & -48 \\
48 & 41 & 34 & 27 & 20 & 13 & 6 & -1 & -8 & -15 & -22 & -29 & -36 & -43 & -50 & -57 & -64 \\
32 & 25 & 18 & 11 & 4 & -3 & -10 & -17 & -24 & -31 & -38 & -45 & -52 & -59 & -66 & -73 & -80 \\
16 & 9 & 2 & -5 & -12 & -19 & -26 & -33 & -40 & -47 & -54 & -61 & -68 & -75 & -82 & -89 & -96 \\
\hline
\end{tabular}

Fig. 2: A rational Dyck path $x \in \mathfrak{D}_{7,16}$.

Theorem 2.9 The map $\gamma$ is a bijection between dominant affine permutations in $\widetilde{\mathfrak{S}}_{n}$ and balanced $n$-flush abaci. The abacus $\gamma(\omega)$ is p-flush if and only if $\omega \in \widetilde{\mathfrak{S}}_{n}^{p}$ is p-stable.

Recall the map $\alpha$ from cores to balanced flush abaci that was introduced in Section 1 . We obtain a bijection $\alpha^{-1} \circ \gamma$ between the dominant affine permutations in $\widetilde{\mathfrak{S}}_{n}$ and $n$-cores. In particular $\alpha^{-1} \circ \gamma$ restricts to a bijection between dominant $p$-stable affine permutations and $\mathfrak{C}_{n, p}$. The following result appears to be new.

Proposition 2.10 Let $\omega \in \widetilde{\mathfrak{S}}_{n}$ be a dominant affine permutation. Then $\alpha^{-1} \circ \gamma\left(\omega^{*}\right)$ is the conjugate partition of $\alpha^{-1} \circ \gamma(\omega)$.

\section{The codinv statistic}

Let $n$ and $p$ be positive coprime integers. A rational Dyck path is a lattice path $x$ that starts at $(0,0)$, consists of $n$ North steps $N=(0,1)$ and $p$ East steps $E=(1,0)$, and never goes below the diagonal with rational slope $n / p$. Denote the set of all rational Dyck paths by $\mathfrak{D}_{n, p}$.

For $(i, j)$ with $0 \leq i \leq p$ and $0 \leq j \leq n$ place the label $\ell_{i, j}=j p-i n$ in the unit square with bottom right corner $(i, j)$. Given a rational Dyck path $x \in \mathfrak{D}_{n, p}$, we assign to each of its steps the label $\ell_{i, j}$, where $(i, j)$ is the starting point of the step. Let $\left(\ell_{1}, \ell_{2}, \ldots, \ell_{n}\right)$ be the vector consisting of the labels of the North steps of $x$ (indicated in red in Figure 2 ordered increasingly. Denote by $H(x)$ the set of positive labels below $x$ (indicated in green in Figure 22. These correspond exactly to those boxes below $x$ but strictly above the diagonal. Hence we have area $(x)=\# H(x)$.

We introduce the following definitions. A codinv pair of $x$ is a pair of integers $(\ell, h)$ such that $\ell$ is the label of a North step of $x$ and $h \in H(x)$ and $\ell \leq h \leq \ell+p$. The codinv tableau of $x$ is the collection of numbers

$$
d_{i, j}(x)=\#\left\{\left(\ell_{i}, h\right): h \in H, \ell_{i}<h<\ell_{i}+p \text { and } h \equiv \ell_{j} \quad \bmod n\right\}
$$

where $i, j \in[n]$ with $i<j$. See Figure 3 for an example.

Similar constructions have appeared before. First we remark that the codinv tableau is related to, albeit not the same as, the laser fillings of Ceballos, Denton and Hanusa [CDH15, Def. 5.13]. The row-sums and column-sums of the codinv tableau and the laser filling of a Dyck path agree. However, the codinv tableau is always of staircase shape while the laser filling sits inside the boxes below the rational Dyck path. 


$\begin{array}{llllllll}d_{1,7} d_{2,7} d_{3,7} d_{4,7} d_{5,7} d_{6,7} & 2 & 3 & 2 & 2 & 2 & 2 \\ d_{1,6} d_{2,6} d_{3,6} d_{4,6} d_{5,6} & & 3 & 2 & 1 & 0 & 0 & \\ d_{1,5} d_{2,5} d_{3,5} d_{4,5} & = & 2 & 2 & 1 & 0 & & \\ d_{1,4} d_{2,4} d_{3,4} & & 2 & 2 & 1 & & \\ d_{1,3} d_{2,3} & 1 & 1 & & & \\ d_{1,2} & & 0 & & & & \end{array}$

Fig. 3: The codinv tableau of the Dyck path in Figure 2.

Secondly we note that codinv pairs have been considered by Gorsky and Mazin [GM13] using slightly different notation. However, they only considered the column-sums of the codinv tableau.

Our next aim is to relate rational Shi tableaux to codinv tableaux. Our starting point is an elegant bijection $\varphi: \mathfrak{D}_{n, p} \rightarrow \mathfrak{C}_{n, p}$ between rational Dyck paths and simultaneous cores discovered by Anderson [And02]. For any finite set $H=\left\{h_{1}, \ldots, h_{k}\right\}$ of positive integers there exists a unique partition $\lambda$ such that $H$ is the set of hook lengths of the cells in the first column of $\lambda$. For any $x \in \mathfrak{D}_{n, p}$ let $\varphi(x)$ be the partition such that the set of hook lengths of the cells in its first column equals $H(x)$. The Dyck path in Figure 2 thereby corresponds to the core in Figure 1

Theorem 3.1 And02, Prop. 1] The map $\varphi: \mathfrak{D}_{n, p} \rightarrow \mathfrak{C}_{n, p}$ is a bijection.

Gorsky, Mazin and Vazirani [GMV14, Sec. 3.1] defined a generalised Anderson map $\mathcal{A}$ that bijectively maps the set of $p$-stable affine permutations to the set of rational parking functions. We are interested in the restriction of the Anderson map to the set of dominant $p$-stable affine permutations, which can be written as the composition $\mathcal{A}=\varphi^{-1} \circ \alpha^{-1} \circ \gamma$ of maps we have already discussed. Here the inverse of Anderson's bijection $\varphi$ makes an appearance, which accounts for the name of the Anderson map. We use the Anderson map to relate rational Shi tableaux to the codinv statistic.

Theorem 3.2 Let $n, p$ be positive coprime integers and $\omega \in \widetilde{\mathfrak{S}}_{n}^{p}$ be a dominant p-stable affine permutation. Then the rational Shi tableau of $\omega$ equals the codinv tableau of $\mathcal{A}(\omega)$. That is, $t_{i, j}^{p}(\omega)=d_{i, j}(\mathcal{A}(\omega))$ for all $i, j \in[n]$ with $i<j$.

The zeta map $\zeta: \mathfrak{D}_{n, p} \rightarrow \mathfrak{D}_{n, p}$ is a map on rational Dyck paths that has appeared many times in the literature. Andrews, Krattenthaler, Orsina and Papi [AKOP02] first defined the inverse of the zeta function in the Catalan case $\mathfrak{D}_{n, n+1}$. Haglund [Hag03] studied the Catalan instance of the zeta map in connection with diagonal harmonics. More recently multiple descriptions of the rational zeta map have been found (see [GM13, ALW15]).

The zeta map can be defined as follows. Let $x=x_{1} x_{2} \ldots x_{n+p} \in \mathfrak{D}_{n, p}$ be a rational Dyck path with steps $x_{i} \in\{N, E\}$. For $i \in[n+p]$ let $\ell\left(x_{i}\right)$ be the label of the step $x_{i}$. Then $\zeta(x)=x_{\sigma(1)} x_{\sigma(2)} \ldots x_{\sigma(n+p)}$, where $\sigma \in \mathfrak{S}_{n+p}$ is the unique permutation such that $\ell\left(x_{\sigma(1)}\right)<\ell\left(x_{\sigma(2)}\right)<\cdots<\ell\left(x_{\sigma(n+p)}\right)$. The zeta map is accompanied by a second map on rational Dyck paths that is called the eta map in [CDH15]. It is defined by $\eta(x)=\zeta \circ \varphi^{-1}\left(\left(\varphi(x)^{\prime}\right)\right.$ where $\lambda^{\prime}$ is the conjugate partition of $\lambda$ and $\varphi$ is Anderson's bijection.

Using the codinv tableau we reprove a connection between the Anderson map, the zeta map and the Pak-Stanley labelling that was already observed in [GMV14, Thm. 5.3]. Note that each rational Dyck 
path $x \in \mathfrak{D}_{n, p}$ is the South-West boundary of a partition $\lambda$ fitting inside the $p \times n$ rectangle. We call $\lambda$ the complement of $x$. For example, the complement of the Dyck path $x$ in Figure 2 is the partition $\lambda=(11,6,6,4,3,2)$.

Theorem 3.3 Let $n, p$ be positive coprime integers and $\omega \in \widetilde{\mathfrak{S}}_{n}^{p}$ be a dominant $p$-stable affine permutation. Then the complement of $\zeta \circ \mathcal{A}(\omega)$ equals the (reversed $\left.{ }^{(i)}\right)$ Pak-Stanley labelling $f(\omega)$. Moreover, the complement of $\eta \circ \mathcal{A}(\omega)$ equals the (reversed) dual Pak-Stanley labelling $f^{*}(\omega)$.

It is well known that $\operatorname{skl}(\varphi(x))=(n-1)(p-1) / 2-\operatorname{area}(\zeta(x))$. This follows from Armstrong's definition of the zeta map [ALW15, Thm 4.8], which uses cores. As a first consequence of Theorem 3.3 we obtain a justification for calling the codinv tableau a refinement of the skew length statistic.

Corollary 3.4 Let $x \in \mathfrak{D}_{n, p}$. Then $\sum_{i, j} d_{i, j}(x)=(n-1)(p-1) / 2-\operatorname{area}(\zeta(x))$.

Furthermore we may now use a result of Ceballos, Denton and Hanusa [CDH15] to prove that each rational Dyck path is determined uniquely by its codinv tableau, or equivalently, that each dominant $p$ stable affine permutation is determined uniquely by its rational Shi tableau.

Theorem 3.5 Let $x, y \in \mathfrak{D}_{n, p}$ such that $d_{i, j}(x)=d_{i, j}(y)$ for all $i, j \in[n]$ with $i<j$. Then $x=y$.

Proof of Theorems 2.8 and 3.5: We deduce the claim from [CDH15, Thm. 6.3], which asserts that any rational Dyck path $x$ can be reconstructed from the pair $(\zeta(x), \eta(x))$. By Theorems 3.2 and 3.3 the codinv tableau of $x$ encodes both $\zeta(x)$ and $\eta(x)$ in terms of column-sums and row-sums. Therefore it contains enough information to determine the path $x$ uniquely. Using the Anderson map again we obtain the analogous statement for rational Shi tableaux and dominant $p$-stable affine permutations.

Note that the exciting recent result due to Williams [Wil15, Cor. 6.4], that the zeta map is bijective, could replace [CDH15, Thm. 6.3] in the proof of Theorem 2.8

\section{Acknowledgements}

The author is grateful to Cesar Ceballos, Jim Haglund, Christian Krattenthaler and Marko Thiel for insightful discussions.

\section{References}

[AHJ14] Drew Armstrong, Christopher R. H. Hanusa, and Brant C. Jones. Results and conjectures on simultaneous core partitions. European J. Combin. 41, pages 205-220, 2014.

[AKOP02] George Andrews, Christian Krattenthaler, Luigi Orsina, and Paolo Papi. ad-nilpotent $\mathfrak{b}$-ideals in $\operatorname{sl}(n)$ having fixed class of nilpotence: Combinatorics and enumeration. Trans. Amer. Math. Soc. 354, pages 3835-3853, 2002.

[ALW15] Drew Armstrong, Nicholas A. Loehr, and Gregory S. Warrington. Sweep maps: A continuous family of sorting algorithms. Adv. Math. 284, pages 159-185, 2015.

(i) In our convention partitions are weakly decreasing while $f(\omega)$ is weakly increasing. 
[And02] Jaclyn Anderson. Partitions which are simultaneously $t_{1}$ - and $t_{2}$-cores. Discrete Math. 248, pages 237-243, 2002.

[BB05] Anders Björner and Francesco Brenti. Combinatorics of Coxeter Groups, volume $231 \mathrm{of} \mathrm{Grad.}$ Texts in Math. Springer, New York, 2005.

[CDH15] Cesar Ceballos, Tom Denton, and Christopher R. H. Hanusa. Combinatorics of the zeta map on rational Dyck paths. 2015. Preprint at arXiv:1504.06383v3.

[FTV11] Susanna Fishel, Eleni Tzanaki, and Monica Vazirani. Counting Shi regions with a fixed separating wall. In Proceedings of the 23rd International Conference on Formal Power Series and Algebraic Combinatorics (FPSAC), Reykjavík, Iceland, pages 351-362, 2011.

[GM13] Eugene Gorsky and Mikhail Mazin. Compactified Jacobians and $q, t$-Catalan numbers, I. J. Combin. Theory Ser. A 120, pages 49-63, 2013.

[GMV14] Eugene Gorsky, Mikhail Mazin, and Monica Vazirani. Affine permutations and rational slope parking functions. 2014. Preprint at arXiv: 1403.0303.

[Hag03] James Haglund. Conjectured statistics for the $q, t$-Catalan numbers. Adv. Math. 175, pages 319-334, 2003.

[Hum90] James E. Humphreys. Reflection Groups and Coxeter Groups, volume 29 of Cambridge Stud. Adv. Math. Cambridge Univ. Press, Cambridge, 1990.

[JK81] Gordon James and Adalbert Kerber. The Representation Theory of the Symmetric Group, volume 16 of Encyclopedia Math. Appl. Addison-Wesley, Reading MA, 1981.

[Las01] Alain Lascoux. Ordering the affine symmetric group. In Proceedings of the Euroconference, Algebraic Combinatorics and Applications (ALCOMA), Gößweinstein, Germany, 1999, pages 219-231. Springer, Berlin, 2001.

[Shi86] Jian-Yi Shi. The Kazhdan-Lusztig Cells in Certain Affine Weyl Groups, volume 1179 of Lecture Notes in Math. Springer, Berlin Heidelberg, 1986.

[Sta96] Richard P. Stanley. Hyperplane arrangements, interval orders, and trees. Proc. Nat. Acad. Sci. 93, pages 2620-2625, 1996.

[Stu10] Christian Stump. $q, t$-Fuß-Catalan numbers for finite reflection groups. J. Algebraic Combin. 32 (1), pages 67-97, 2010.

[Sul15] Robin Sulzgruber. Rational Shi tableaux and the skew length statistic. 2015. Preprint at arXiv:1512.04320.

[Thi15] Marko Thiel. From Anderson to zeta. 2015. Preprint at arXiv:1504.07363v3.

[Wil15] Nathan Williams. Sweeping up zeta. 2015. Preprint at arXiv:1512.01483v2.

[Xin15] Guoce Xin. Rank complement of rational Dyck paths and conjugation of $(m, n)$-core partitions. 2015. Preprint at arXiv:1504.02075v2. 